\title{
Direct Coulomb and Phonon-Mediated Coupling between Spatially Separated Electron Gases
}

\author{
H. C. Tso and P. Vasilopoulos \\ Department of Physics, Concordia University, 1455 de Maisonneuve Blvd. West, Montréal, Québec, Canada H3G IM8 \\ F. M. Peeters \\ Department of Physics, University of Antwerp (UIA), B-2610 Antwerp, Belgium
}

(Received 13 November 1991)

\begin{abstract}
Momentum transfer due to electron-electron interaction between two quasi-two-dimensional electron gases, whose edges are separated by a distance $d$, is studied at low temperatures $(T)$. The coupling between the gases results from the direct Coulomb (screened) interaction and from the interaction mediated by virtual phonon exchange. The former has a $T^{2}$ dependence and varies approximately as $d^{-4}$ for large $d$. The latter depends very weakly on $d$ and, combined with the former, accounts extremely well for the $T$ and $d$ dependences of the relaxation time as measured by Gramila et al. [Phys. Rev. Lett. 66, $1216(1991)]$.
\end{abstract}

PACS numbers: 73.20.Dx

Coulomb coupling between two spatially separated electron-gas layers has been predicted to influence the transport properties of the individual gases $[1,2]$. This has been confirmed recently by the observation [3] of a contactless current in a semi-infinite three-dimensional (3D) layer when an electric field was applied to a quasitwo-dimensional electron gas (Q2DEG) $300 \AA$ apart and vice versa. Very recently the coupling between two Q2DEG's, forming a symmetric double quantum well, was investigated [4,5]. When no current is allowed to flow in the drag well, a voltage [4,5] is developed from the accumulation of charges swept along the direction of the electric field in the drag well; it is opposite to the resistive voltage in the drive well and balances the drag due to the interlayer interactions. Theoretical calculations $[4,6]$ of the direct Coulomb scattering, with dynamic screening, between electrons of two Q2DEG's, with centers separated by a distance $a$, found that the induced voltage should have an $a^{-4}$ and a $T^{2}$ dependence. The observation of appreciable deviations from a $T^{2}$ dependence [4] and the weak spacing dependence of these deviations [5] led to the consideration of real [4] or virtual [5] phonons as a second coupling mechanism. However, no calculations were presented and real phonon exchange was estimated [4] to be too weak to account for the observations. As a result the second coupling mechanism, though considered, remained unidentified. In this Letter we show that virtual phonon exchange between electrons in the two wells leads to an important additional coupling that depends very weakly on $a$. When combined with the direct Coulomb coupling, it accounts extremely well for the experimental observations [4,5]. At the same time, we show that exchange of real phonons derived from the usual "incoherent" electron-phonon scattering cannot account for the observation.

We model the system with infinitely deep quantum wells of width $b$ and with their centers separated by a distance $a$. The many-body Hamiltonian describing this system is

$$
H(t)=H_{a}(t)+H_{\beta}(t)+H_{\alpha \beta}(t)+H_{p}(t)+H_{i}(t) .
$$

Here $H_{\alpha}$ is the Hamiltonian of the electrons in the drive well $(\alpha)$ and contains electron-electron, electron-impurity, and electron-phonon interactions; the same holds for $H_{\beta}$, the Hamiltonian of the electrons in the drag well $(\beta)$. $H_{a \beta}$ is the Coulomb interaction between electrons in the two wells, $H_{p}$ is the phonon Hamiltonian, and $H_{i}$ the impurity Hamiltonian. Tunneling between wells and the vertex corrections of the three-point vertex function [see Eq. (149) of Ref. [7]] are neglected for the confined degenerate electron gases. However, the vertex correction of the four-point vertex function [see Eq. (144) of Ref. [7]] responsible for the coupling of electrons in different wells will be retained in our calculation. The equation of motion of the thermodynamic Green's function leads [7,8] to the following coupled equations:

$$
\begin{aligned}
& -\frac{m^{*}}{e} \frac{\partial \mathrm{j}_{a}}{\partial t}+e n_{\alpha} \mathrm{E}=\mathrm{F}^{\alpha \beta}+\mathrm{F}_{p}^{\alpha \beta}, \\
& -e n_{\beta} \frac{V_{D}}{L} \frac{\mathrm{j}_{\beta}}{j_{\beta}}-\frac{m^{*}}{e} \frac{\partial \mathrm{j}_{\beta}}{\partial t}=\mathrm{F}^{\beta \alpha}+\mathrm{F}_{p}^{\beta a} .
\end{aligned}
$$

Here, $V_{D}$ is the measured drag voltage, $L$ the length of the specimen, $n_{\alpha}\left(n_{\beta}\right)$ the 2D density of the drive (drag) well, $j_{\alpha}\left(j_{\beta}\right)$ the corresponding 2D current density, and E the electric field applied only in the drive well. $\mathbf{F}_{p}^{\alpha \beta}$ is the frictional force due to the phonon-mediated interaction; the frictional force due to direct Coulomb, phonon, and impurity scatterings [9] is

$$
\mathbf{F}^{\alpha \beta} \equiv \int d z_{1} \int_{t_{0}}^{t_{1}} d(3)\left[\Pi_{>}^{\alpha} \nabla_{1} \tilde{v}_{\alpha \beta}>-\Pi_{<}^{\alpha} \nabla_{1} \tilde{v}_{\alpha \beta}<\right]
$$

expressed in terms of $\Pi_{\gtrless}^{\alpha} \equiv \Pi_{\gtrless}^{\alpha}(1 ; 3)$, the nonequilibrium polarizability, and $\tilde{v}_{\alpha \beta} \equiv \tilde{v}_{\alpha \beta}(1 ; 3)$, the nonequilibrium screened interaction between electrons in the $\alpha$ well and the medium (phonons and impurities included). The arguments in $\Pi^{\alpha}(1 ; 2)$ and $\tilde{v}_{\alpha \beta}(1 ; 2)$ represent the correlation between the space-time coordinates $\left(\mathbf{x}_{1}, t_{1}\right)$ and $\left(\mathbf{x}_{2}, t_{2}\right)$ and $\int_{t_{0}}^{t_{1}} d(3)$ is a shorthand for $\int d \mathbf{x}_{3} \int_{t_{0}}^{t_{1}} d t_{3}$. The appearance of the drag voltage $V_{D}$ in Eq. (3) is due to the accumulation of electrons at one end of the drag well. If 
the system is uniform, $V_{D}$ vanishes identically.

For weak electric fields, we may write $\mathbf{j}_{\alpha, \beta}=n_{\alpha, \beta} e \mathbf{v}_{d}^{\alpha, \beta}$ with $\mathbf{v}_{d}^{\alpha}\left(\mathbf{v}_{d}^{\beta}\right)$ the corresponding drift velocities. Then the forces can be linearized over the drift velocities giving $\mathbf{F}^{\alpha \beta}=\Omega_{\alpha \beta}^{c}\left(\mathbf{v}_{d}^{\alpha}-\mathbf{v}_{d}^{\beta}\right)+\left(\Omega_{\alpha i}+\Omega_{\alpha p}\right) \mathbf{v}_{d}^{\alpha}$ and $\mathbf{F}_{p}^{\alpha \beta}=\Omega_{\alpha \beta}^{p}\left(\mathbf{v}_{d}^{\alpha}\right.$ $\left.-\mathbf{v}_{d}^{\beta}\right)$. Here $\Omega_{\alpha \beta}^{c}, \Omega_{\alpha p}, \Omega_{\alpha i}$, and $\Omega_{\alpha \beta}^{p}$ are the relaxation frequencies per unit area due to the direct Coulomb coupling, phonon scattering, impurity scattering, and the phonon-mediated electron-electron coupling, respectively. In the steady state and in the absence of the drag current $\left(t_{0}=-\infty, j_{\beta}=0\right)$, Eq. (3) gives $-e n_{\beta} V_{D} / L=\left(\Omega_{\alpha \beta}^{c}\right.$ $\left.+\Omega_{\alpha \beta}^{p}\right)_{v d}^{\alpha}$. Physically, $\mathbf{F}_{c}^{\alpha \beta}$ is the frictional force without consideration of the coupling vertex between electrons in different wells obtained from the self-energy of electrons ( $\alpha$ well) given in Fig. $1(a)$ and $F_{p}^{\alpha \beta}$ is the frictional force due to the coupling vertex. Since $V_{D} / v_{d}^{\alpha}$ depends only on $\boldsymbol{\Omega}_{\boldsymbol{\alpha} \beta}^{\boldsymbol{c}}$ and $\boldsymbol{\Omega}_{\boldsymbol{\alpha} \beta}^{\boldsymbol{p}}$ but not on the incoherent electron-phonon coupling nor on the electron-impurity coupling, we consider the Fourier component of the effective interaction without impurity scattering [see Eq. (87) of Ref. [7]]. We then have

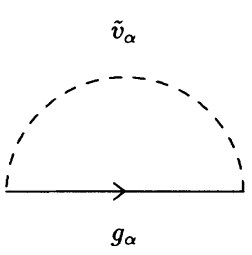

(a)

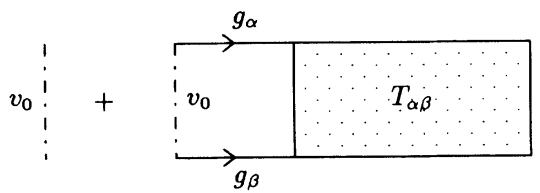

(c)

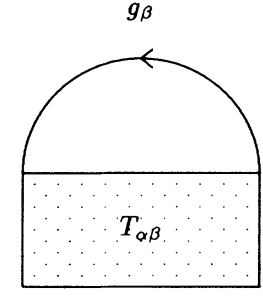

(b)

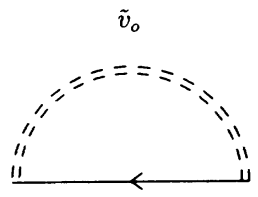

$g_{\beta}$

(d)
FIG. 1. (a) Self-energy diagram of electrons ( $\alpha$ well) due to mutual Coulomb scattering; (b) self-energy diagram of electrons ( $\alpha$ well) due to exchange of virtual phonons; (c) multiple scattering of the coupling vertex; (d) modified self-energy diagram of electrons ( $\alpha$ well) due to exchange of virtual phonons.

$$
\tilde{v}_{a \beta}\left(\mathbf{q}_{\|} ; \omega ; t\right)=F\left(q_{\|}\right) \frac{2 \pi e^{2} e^{-\left|\mathbf{q}_{\|}\right| a}}{\left|\mathbf{q}_{\|}\right| \epsilon\left(\mathbf{q}_{\|} ; \omega ; t\right)}+\sum_{q_{z}}\left|I\left(-i q_{z}\right)\right|^{2} \frac{\left|M^{\prime}(\mathbf{q} ; \omega)\right|^{2}}{\left|\epsilon\left(\mathbf{q}_{\|} ; \omega ; t\right)\right|^{2}} D_{\lambda}(\mathbf{q} ; \omega)
$$

with $\left(\zeta=q_{\|} b\right)$

$$
\begin{aligned}
& F\left(q_{\|}\right)=\frac{1}{\zeta^{2}+4 \pi^{2}}\left(3 \zeta+\frac{8 \pi^{2}}{\zeta}-\frac{32 \pi^{4}}{\zeta^{2}} \frac{1}{\zeta^{2}+4 \pi^{2}}\left(1-e^{-\zeta}\right)\right), \\
& M^{\prime}(\mathbf{q} ; \omega)=M\left(\mathbf{q} ; \omega_{\lambda}\right)\left(1-\frac{2 \pi e^{2}}{\left|\mathbf{q}_{\|}\right|} F\left(q_{\|}\right)\left(1+e^{-\left|q_{\|}\right| a}\right) \Pi^{\beta(0)}\left(\mathbf{q}_{\|} ; \omega\right)\right),
\end{aligned}
$$

and the nonequilibrium dielectric function

$$
\epsilon\left(\mathbf{q}_{\| ;} ; \omega t\right)=\left(1-\frac{2 \pi e^{2}}{\left|\mathbf{q}_{\|}\right|} F\left(q_{\|}\right) \Pi^{\alpha}\left(\mathbf{q}_{\|} ; \omega ; t\right)\right)\left(1-\frac{2 \pi e^{2}}{\left|\mathbf{q}_{\|}\right|} F\left(q_{\|}\right) \Pi^{\beta}\left(\mathbf{q}_{\|} ; \omega ; t\right)\right)-\left(\frac{2 \pi e^{2}}{\left|\mathbf{q}_{\|}\right|}\right)^{2} e^{-2\left|\mathbf{q}_{\|}\right| a}\left|F\left(q_{\|}\right)\right|^{2} \Pi^{\alpha}\left(\mathbf{q}_{\|} ; \omega ; t\right) \Pi^{\beta}\left(\mathbf{q}_{\|} ; \omega ; t\right) \text {. }
$$

Here $\Pi^{\alpha, \beta(0)}$ is the equilibrium polarizability of electrons, $D_{\lambda}(\mathbf{q} ; \omega)$ is the dressed phonon propagator, $M\left(\mathbf{q} ; \omega_{\lambda}\right)$ is the matrix element of the electron-acoustic phonon interaction, and $\omega_{\lambda}$ is the phonon frequency. The first term on the right-hand side of Eq. (5) is the screened Coulomb potential, whereas the second term is the phonon-mediated interaction which gives rise to the ordinary "incoherent phonon scattering" and the phonon-mediated electron-electron interaction. If only the direct Coulomb scattering is considered and vertex corrections are neglected, we obtain, in the random-phase approximation (RPA), $\boldsymbol{\Omega}_{\alpha \beta}^{c}$ as

$$
\Omega_{\alpha \beta}^{c}=-\left.\frac{2 \hbar}{m^{*}} \int_{-\infty}^{\infty} \frac{d \omega}{2 \pi} \sum_{\mathbf{q}_{\|}} \frac{\left|F\left(q_{\|}\right)_{v}\left(q_{\|}\right)\right|^{2}}{\left|\epsilon_{+}\left(\mathbf{q}_{\|} ; \omega\right)\right|^{2}} q_{\|}^{2} \frac{\partial n\left(\omega^{\prime}\right)}{\partial \omega^{\prime}}\right|_{\omega^{\prime}=-\omega} \operatorname{Im}\left[\Pi_{+}^{q(0)}\right] \operatorname{Im}\left[\Pi_{+}^{\beta(0)}\right]
$$

with $\Pi_{+}^{q(0)} \equiv \Pi_{+}^{q(0)}\left(\mathbf{q}_{\|} ; \omega\right), v\left(q_{\|}\right)=2 \pi e^{2} / q_{\|}$, and $\epsilon_{+}\left(\mathbf{q}_{\|} ; \omega\right)$ the equilibrium counterpart of $\epsilon\left(\mathbf{q}_{\|} ; \omega+i 0^{+} ; \infty\right)$. For small $T$, it can be shown that $\Omega_{a \beta}^{c}$ behaves quadratically with $T$ as shown in Fig. 2. The solid, dashed, and dotted lines correspond to the calculated $\Omega_{\alpha \beta}^{c}$ at separations of 175,225 , and $550 \AA$, respectively. It behaves approximately as $d^{-4}$ at constant $T$. However, electrons in the $\alpha$ well can exchange real and virtual phonons with electrons in the $\beta$ well. This leads to additional electron-electron couplings. The exchange of real phonons has an extremely small effect on the coupling compared to that involving virtual phonons because it is proportional to $\left|M\left(\mathbf{q} ; \omega_{\lambda}\right)\right|^{4}$ as opposed to $\left|M\left(\mathbf{q} ; \omega_{\lambda}\right)\right|^{2}$ for virtual phonons. The extra factor of $\left|M\left(\mathbf{q} ; \omega_{\lambda}\right)\right|^{2}$ comes directly from the nonequilibrium propagator Isee Eq. (127) of Ref. [7]]. For simplicity, we employ the jellium model for the lattice so that $\left|M^{\prime}\left(\mathbf{q} ; \omega_{\lambda}\right)\right|^{2}=2 \pi e^{2} \omega_{\lambda} /|\mathbf{q}|^{2}$. As for the dressed phonon propagator, the static limit of the polarizations due to the electrons in both wells will be adopted here since electrons can follow exactly the vibration of the acoustic phonons. In this case, the dressed phonon propagator [7] 
is given by $D_{\lambda}(\mathbf{q} ; \omega)=2 \omega_{\lambda} /\left(\omega^{2}-\tilde{\omega}_{\lambda}^{2}\right)$, the modified phonon modes $\tilde{\omega}_{\lambda}$ by

$$
\tilde{\omega}_{\lambda}^{2}=\omega_{\lambda}^{2}-2 \omega_{\lambda} \sum_{q_{z}} \frac{\left|M_{p}^{e}(\mathbf{q})\right|^{2}\left|I\left(-i k_{z}\right)\right|^{2}}{\epsilon_{+}\left(\mathbf{q}_{\|} ; 0\right)}\left[\Pi_{\alpha}\left(\mathbf{q}_{\|} ; 0\right)+\Pi_{\beta}\left(\mathbf{q}_{\|} ; 0\right)\right],
$$

the (virtual) phonon-mediated interaction $v_{0}$ by

$$
{ }^{v_{0}}\left(\mathbf{q}_{\|}, \omega\right)=\sum_{q_{z}}\left|I\left(-i q_{z}\right)\right|^{2}\left|M_{p}^{e}(\mathbf{q})\right|^{2} /\left|\epsilon_{+}\left(\mathbf{q}_{\|} ; 0\right)\right|^{2}\left[2 \omega_{\lambda} /\left(\omega^{2}-\tilde{\omega}_{\lambda}^{2}\right)\right],
$$

and the form factor by

$$
I\left(-i q_{z}\right)=8 \pi^{2} \sin \left(q_{z} b / 2\right) / q_{z} b\left(q_{z}^{2} b^{2}-4 \pi^{2}\right) .
$$

For very thin wells $(b \rightarrow 0)$, we have

$$
J=\sum_{q_{z}} 4 \pi e^{2}\left|I\left(-i q_{z}\right)\right|^{2} /|\mathbf{q}|^{2} \rightarrow 2 \pi e^{2} /\left|\mathbf{q}_{\|}\right| .
$$

For finite $b, J$ reduces to $[1+N(\zeta)] 2 \pi e^{2} /\left|\mathbf{q}_{\|}\right|$. The extra factor $N(\zeta)$ depends on the detailed structure of the wave function of electrons in the wells. For infinitely deep quantum wells, $N(\zeta)=\left\{8 \pi^{2} \sinh (\zeta / 2) /\left[\zeta\left(\zeta^{2}+4 \pi^{2}\right)\right]\right\}^{2}-1$ and it is very small compared to one for thin wells. This is also the case when $b$ is finite and $q_{z} \rightarrow 0$, i.e., for long wavelength (exchanged) phonons [10]. To reduce the numerical work, we use the thin-well approximation and the jellium model for $v_{0}$. To understand the validity of the latter, we compute $M_{p}^{e}$ from first principles using the monopole term of the electron-ion interaction instead of the usual parametrized interaction $\left|M_{p}^{e}(\mathbf{q})\right|^{2}=D^{2} q / 2 \rho v_{s}$ since $D$, the deformation potential, is the Fourier transform of the electron-ion interaction [11] times the number density of the lattice $N$. In other words, $\left|M_{p}^{e}(q)\right|^{2}$ $=8\left(\pi e^{2} N\right)^{2} / q^{3} \rho v_{s}$. Here $\rho$ is the lattice density and $v_{s}$ the speed of sound in GaAs. With this, Eq. (10) becomes

$$
\tilde{\omega}_{\lambda}^{2}=\omega_{\lambda}^{2}-\omega_{0}^{2}\left[\Pi_{\alpha}\left(\mathbf{q}_{\|} ; 0\right)+\Pi_{\beta}\left(\mathbf{q}_{\|} ; 0\right)\right] 2 \pi e^{2} / q_{\|} \epsilon_{+}\left(\mathbf{q}_{\|} ; 0\right),
$$
where $\omega_{0}^{2}=4 \pi e^{2} N^{2} / \rho$. With $\rho=5.36 \mathrm{~g} / \mathrm{cm}^{3}$ and $N$

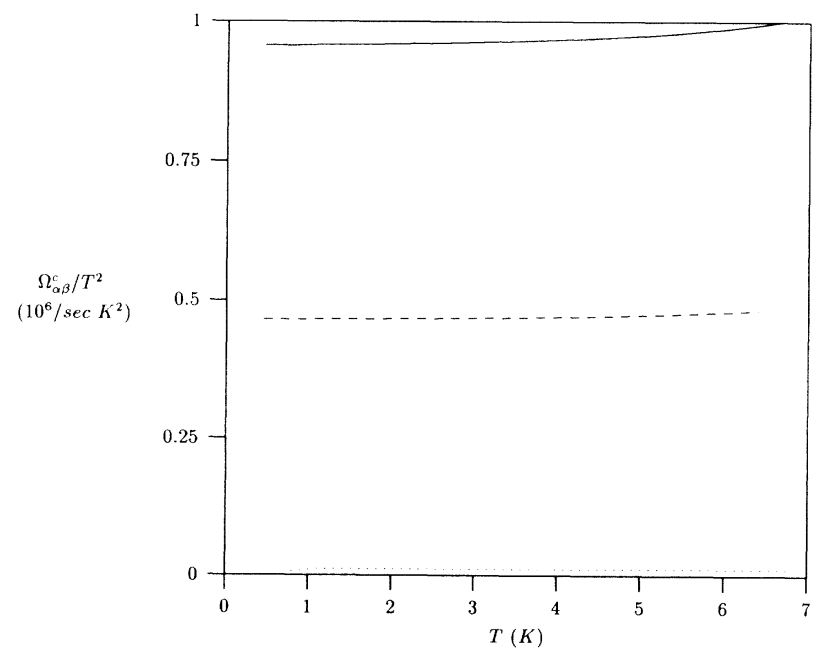

FIG. 2. The relaxation frequency per temperature squared (per unit area) due to mutual Coulomb scattering $\boldsymbol{\Omega}_{\alpha \beta}^{c}$ as a function of temperature at separations of $175 \AA$ (solid line), $225 \AA$ (dashed line), and $500 \AA$ (dotted line). The $2 \mathrm{D}$ electron density is $1.5 \times 10^{11} / \mathrm{cm}^{2}$.
$-4.5 \times 10^{22} \mathrm{~cm}^{-3}$ for GaAs, $\omega_{0} \sim 10^{12} \mathrm{sec}^{-1}$. Because of the Fermi factors in $\Omega_{\alpha \beta}^{p}$ [cf. Eq. (13)] and the factor $\omega^{2}-\tilde{\omega}_{\lambda}^{2}$ in $v_{0}$, the dominant contribution to $\Omega_{\alpha \beta}^{p}$ comes from $\omega \sim k_{B} T \sim \omega_{\lambda}$ and $k_{B} T / \hbar \sim 7 \times 10^{11} \mathrm{sec}^{-1} \sim \omega_{0}$ at $T \sim 5 \mathrm{~K}$. When $\omega_{\lambda}=\omega_{0}$ (jellium model), $\omega_{\lambda}^{2}$ will further reduce to $\tilde{\omega}_{\lambda}^{2}=\omega_{\lambda}^{2} / \epsilon_{+}\left(q_{\| ;} ; 0\right)$, and

$$
{ }^{\prime}{ }_{0}\left(\mathbf{q}_{\|} ; \omega\right) \simeq \frac{2 \pi e^{2}}{\left|\mathbf{q}_{\|}\right| \epsilon_{+}\left(\mathbf{q}_{\|} ; 0\right)} \frac{\tilde{\omega}_{\lambda}^{2}}{\omega^{2}-\tilde{\omega}_{\lambda}^{2}} .
$$

The separation dependence of $v_{0}$ is through $\epsilon_{+}\left(q_{\|} ; 0\right)$ and is very weak. The deviations $\Delta v^{\prime}{ }_{0}$ from this value of $v^{\prime}$, obtained in the jellium model for very thin wells, are given by $\Delta v_{0}=f_{c}(q, \omega)_{v_{0}}$ with $f_{c}(q, \omega)$ depending weakly on $q$ and $\omega$. For simplicity, we take $f_{c}$ constant and use for the total $v_{0}$ the value above multiplied by $1+f_{c}$.

Because of $\omega \sim k_{B} T \sim \tilde{\omega}_{\lambda}$, vertex corrections have to be taken into account [Eq. (138) of Ref. [7]]. The additional contribution to the self-energy of electrons in the drive well $(\alpha)$ due to this effect is illustrated [8,11] in Fig. 1(b). The coupling-vertex $T_{\alpha \beta}$ involves multiple scattering between electrons in the two wells as shown in Fig.

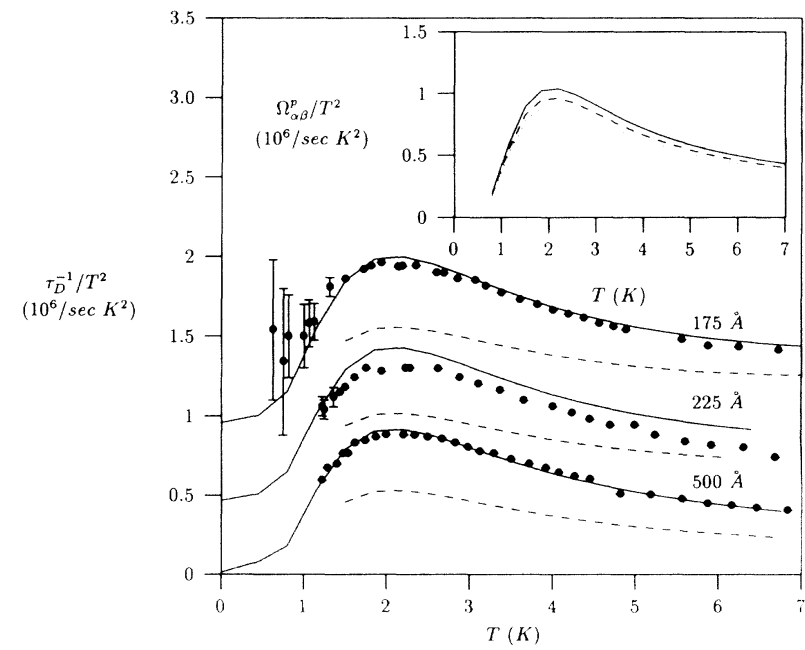

FIG. 3. The relaxation frequency per temperature squared (per unit area) due to both mutual Coulomb scattering and exchange of virtual phonons $\tau_{D}^{-1 /} T^{2}$ as function of temperature at different separations. The 2D electron density is $1.5 \times 10^{11} / \mathrm{cm}^{2}$ and the parameter $f_{c}$ is 0.053 and 0 for the solid and dashed curves, respectively. The solid circles are the experimental results of Ref. [4]. Inset: Contribution to $\tau_{D}^{-1} / T^{2}$ due to exchange of virtual phonons $\Omega_{\alpha \beta}^{p} / T^{2}$ as a function of temperature. The solid, dashed, and dotted curves correspond to separations of 175,225 , and $500 \AA$, respectively. 


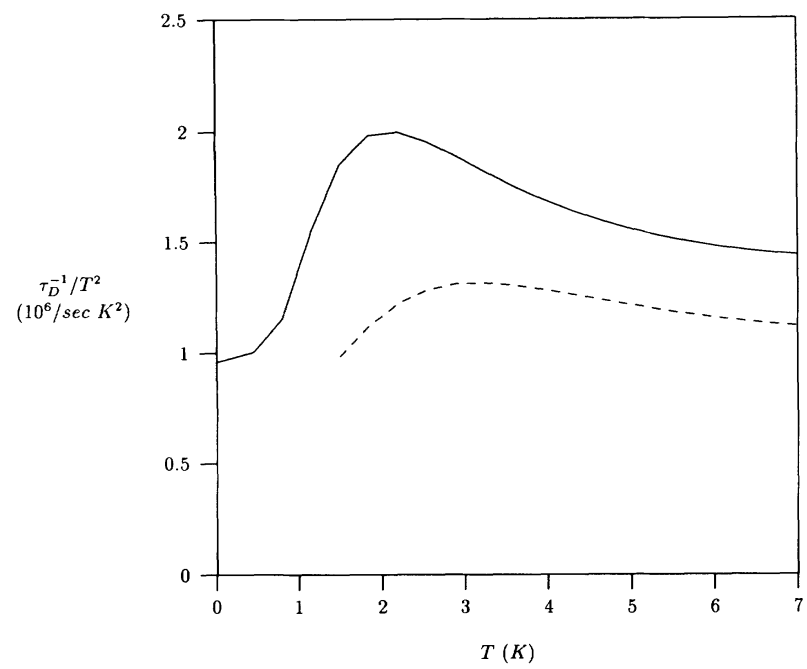

FIG. 4. $\tau_{D}^{-1 / T} T^{2}$ as a function of temperature for $d=175 \AA$. The solid curve is for $n_{\alpha}=n_{\beta}=1.5 \times 10^{11} / \mathrm{cm}^{2}$ and the dashed one is for the same $n_{\alpha}$ and $n_{\beta}=2 n_{\alpha} / 3$.

1(c). For simplicity, we approximate Eq. (11) by

$$
v_{0}\left(\mathbf{q}_{\|} ; \omega\right)=-2 \pi e^{2}\left(1+f_{c}\right) \eta_{+}\left(\tilde{\omega}_{\lambda}-\omega\right) /\left|\mathbf{q}_{\|}\right| \epsilon_{+}\left(\mathbf{q}_{\|} ; 0\right)
$$

[ $\eta_{+}(x)$ is the Heaviside step function], and we further as- sume that $v_{0}$ is a slowly varying function of $q_{\|}$since $q_{\|}$, proportional to the momentum transfer, is very small compared to the screening wave vector. With these approximations, $\quad T_{a \beta}(1,2 ; 3,4) \simeq \tilde{v}_{0}(1 ; 3) \delta(1 ; 2) \delta(3 ; 4)$, and therefore the additional self-energy of the electrons in the drive well due to the virtual phonon exchange, illustrated in Fig. 1(b), becomes Fig. 1(d) with

$$
\tilde{v}_{0}\left(P_{\|} ; \omega\right)=\frac{2 \pi \hbar^{2}}{m^{*}} \frac{\xi_{0}}{\omega-\xi_{0}-\hbar\left|\mathbf{P}_{\|}\right|^{2} / 4 m^{*}}
$$

and $\xi_{0}=\tilde{\omega}_{\lambda} \exp \left[-\hbar^{2} v_{0}(0 ; 0) / m^{*} e^{2}\right]$. In Eq. (12), $\mathbf{P}_{\|}$is the total momentum of two electrons in both wells involved in multiple scattering. In general, $\tilde{v}_{0}$ is very small compared to the Coulomb interaction except at the energy range when $\omega$ is in the neighborhood of $\xi_{0}$ and $P_{\|}$is small. In this range, we have extremely strong phononmediated interaction comparable to the Coulomb interaction. Only those electrons moving opposite to each other $\left(P_{\|}=p_{1}+p_{2} \rightarrow 0\right)$ with more or less the same value of momentum will contribute to this coupling significantly. On the other hand, this phonon-mediated coupling will be very small if the electron densities in the wells are different since only electrons near the Fermi level will take part in multiple scattering and $\mathbf{P}_{\|} \simeq \mathbf{p}_{1 F}+\mathbf{p}_{2 F} \neq 0$. Thus, the contribution to the relaxation frequency is

$$
\Omega_{\alpha \beta}^{p} \simeq-\left.\frac{\hbar}{m^{*}} \int_{-\infty}^{\infty} \frac{d \omega}{2 \pi} \sum_{\mathbf{q}_{1}}\left|\tilde{v}_{0}+\left(P_{0} ; \omega\right)\right|^{2} q_{\|}^{2} \frac{\partial n\left(\omega^{\prime}\right)}{\partial \omega^{\prime}}\right|_{\omega^{\prime}=-\omega} \operatorname{Im}\left[\Pi_{+}^{\alpha(0)}\right] \operatorname{Im}\left[\Pi_{+}^{\beta(0)}\right],
$$

890123 (P.V., F.M.P.), and by the National Science $\left.+i 0^{+}\right) ; \epsilon_{F}^{\alpha}$ and $\epsilon_{F}^{\beta}$ are the corresponding Fermi energies of electrons in the two wells. For $\epsilon_{F}^{\alpha}=\epsilon_{F}^{\beta}, \Omega_{\alpha \beta}^{p} / T^{2}$ is almost independent of the separation and it exhibits a maximum as shown in the inset of Fig. 3. Similar behavior is also observed in Ref. [12]. In Fig. 3, we plot $\left(\Omega_{a \beta}^{c}+\Omega_{\alpha \beta}^{p}\right) / T^{2}$ versus the temperature for $f_{c}=0$ (dashed curves) and $f_{c}=0.053$ (solid curves) at different separations; the latter are in excellent agreement with the experimental results $[4,5]$ indicated by solid circles. For $\epsilon_{F}^{a} \neq \epsilon_{F}^{\beta}$, i.e., for different densities the maximum diminishes as shown in Fig. 4. This is due to the fact that electrons in the two wells which take part in multiple scattering and have energies close to the respective Fermi levels cannot have the same magnitude in momentum $\left(P_{\|} \neq 0\right)$. Thus, contributions from $\Omega_{a \beta}^{p}$ will be smaller because of the broadening of $\tilde{v}_{0}$.

In summary, we have shown that the reported $[4,5]$ deviations of separation and temperature dependences of the Coulomb coupling between two separated quantum wells from those calculated earlier $[4,6]$ can be well accounted for by inclusion of virtual phonon exchange between electrons in the two wells. Real phonon exchange is too small to account for these deviations.

We thank Dr. A. MacDonald for discussions on the virtual phonon exchange. This work was supported by NSERC Grant No. URF0116877 (H.C.T., P.V.), by the Collaborative Research Grant, NATO: 5-2-05/RG No.

[1] P. J. Price, Physica (Amsterdam) 117B, 750 (1983).

[2] I. I. Boiko and Yu M. Sirenko, Phys. Status Solidi 159, 805 (1990).

[3] P. M. Solomon, P. J. Price, D. J. Frank, and D. C. La Tulipe, Phys. Rev. Lett. 63, 2508 (1989).

[4] T. J. Gramila, J. Eisenstein, A. H. MacDonald, L. N. Pfeiffer, and K. W. West, Phys. Rev. Lett. 66, 1216 (1991).

[5] T. J. Gramila, J. Eisenstein, A. H. MacDonald, L. N. Pfeiffer, and K. W. West, Surf. Sci. (to be published).

[6] P. Vasilopoulos and H. C. Tso, in Proceedings of CAPNSERC Workshop on Excitations in Superlattices and Multi-Quantum Wells, Canada, 1991 (Springer-Verlag, Berlin, to be published).

[7] H. C. Tso and N. J. M. Horing, Phys. Rev. B 44, 8886 (1991).

[8] L. P. Kadanoff and G. Baym, Quantum Statistical Mechanics (Benjamin, New York, 1962).

[9] H. C. Tso and P. Vasilopoulos, Phys. Rev. B 44, 12952 (1991).

[10] A. MacDonald (private communication).

[11] G. D. Mahan, Many-Particle Physics (Plenum, New York, 1981).

[12] H. C. Tso and N. J. M. Horing, Phys. Lett. A 152, 498 (1991). 